Article

\title{
A mathematical modeling approach for targeted radionuclide and chimeric antigen receptor- $T$ cell combination therapy
}

\author{
V. Adhikarla ${ }^{*}$, D. Awuah ${ }^{2}$, A. Brummer ${ }^{1}$, E. Caserta $^{3}$, A. Krishnan ${ }^{2}$, F. Pichiorri ${ }^{3}$, M. Minnix ${ }^{4}$, J. Shively ${ }^{4}$, J. Wong5, \\ X. Wang ${ }^{2}$, R. Rockne ${ }^{1 *}$
}

Division of Mathematical Oncology, Department of Computational and Quantitative Medicine, Beckman Research Institute, City of Hope National Medical Center, Duarte, California, USA;

2 Department of Hematology \& Hematopoietic Cell Transplantation, Beckman Research Institute, City of Hope National Medical Center, Duarte, California, USA;

3 Department of Hematologic Malignancies Translational Science, Beckman Research Institute, City of Hope National Medical Center, Duarte, California, USA;

4 Department of Molecular Imaging and Therapy, City of Hope National Medical Center, Duarte, California, USA;

5 Department of Radiation Oncology, City of Hope National Medical Center, Duarte, California, USA;

* Correspondence: R.R. rrockne@coh.org, V.A. vadhikarla@coh.org

Simple Summary: Targeted Radionuclide Therapy (TRT) and immunotherapy - an example being chimeric antigen receptor (CAR)-T cells, represent two potent means of eradicating systemic cancers. While each one as a monotherapy might have limited effect, the potency can be increased with a combination of the two therapies. The complications involved in dosing and scheduling of these therapies make mathematical modeling of these therapies a suitable solution for designing combination treatment approaches. Here we investigate a mathematical model for TRT and CAR-T cell combination therapies. Through analysis of the mathematical model, we find that tumor proliferation rate is the most important factor affecting the scheduling TRT and CAR-T cell treatments, with faster proliferating tumors requiring a shorter interval between the two therapies.

\begin{abstract}
Targeted radionuclide therapy (TRT) has recently seen a surge in popularity, with the use of radionuclides conjugated to small molecules and antibodies. Similarly, immunotherapy also has shown promising results - an example being chimeric antigen receptor (CAR) T-cells therapy in hematologic malignancies. Moreover, TRT and CAR T therapies possess unique features that require special consideration when determining how to dose, time, and sequence combination treatments, including the distribution of TRT dose in the body, the decay rate of the radionuclide, and the proliferation and persistence of the CAR-T cells. These characteristics complicate additive or synergistic effects of combination therapies and warrant a mathematical treatment which includes these dynamics in relation to the proliferation and clearance rates of the target tumor cells. Here we combine two previously published mathematical models in a multiple myeloma setting to explore the effects of dose, timing, and sequencing of TRT and CAR-T cell based therapies. We find that for a fixed TRT and CAR-T cell dose, the tumor proliferation rate is the most important parameter in determining the best timing of TRT and CAR T therapies.
\end{abstract}

Keywords: CAR T; Targeted Radionuclide Therapy; TRT; Mathematical Model; Multiple Myeloma; Immunotherapy; Daratumumab; CS1; Combination Therapy

\section{Introduction}

Immunotherapy has now established itself as one of the advanced treatment options in cancer care including metastatic stages or adjuvant or neoadjuvant settings in many cancers [1]. Immunotherapy attempts to cure cancer by stimulating, arming, and priming the host body's immune system against the tumor. However, patients on immunotherapy also suffer from immune-related adverse events that limit the dose of the therapeutic 
agent administered to the patient. Thus, to boost the efficacy against cancer tumors, immunotherapy may be combined with other established forms of therapy such as radiation. Several active clinical trials are underway to explore the combination of external beam radiation therapy (EBRT) with immunotherapy [2,3] for improved survival and toxicity control.

Chimeric Antigen Receptor T-cell (CAR-T) therapy [4, 5] is a form of immunotherapy where the $\mathrm{T}$ cells are acquired from the patient and are genetically engineered to express a chimeric antigen receptor(s) (CAR) on their surface (Figure 1A). Patient T cells can be engineered to express multiple CARs that can be used to target multiple antigens, which when injected back into the patient target and eradicate the tumor cells based on the antigen expressed. With optimal CAR design and manufacturing, these CAR-T cells further proliferate, and survive (persist) in the body resulting in increased targeting of the tumor cells.

Targeted Radionuclide Therapy (TRT) is a form of radiation therapy where a radionuclide is tagged with another molecule and is injected into the body [6, 7]. The affinity of the attached molecule to tumor cells is exploited to preferentially localize the radiotherapeutic molecule to the target cells. The radionuclide half-life is chosen to best match the kinetics of the molecule targeting cancer cells, for example the pharmacokinetic profile of a small molecule or antibody (Figure 1A). Based on the kinetics of the radiotherapeutic and the type and energy deposited by the radionuclide decay, absorbed dose to the cancer tumor can be determined. The advantage of targeted radionuclide therapy lies in the selectivity of delivering radiation to the cancer cells at the molecular level. Thus, in lieu of external beam radiation therapy, targeted radionuclide therapy is attractive to combine with immunotherapies. In addition, the uptake and thus the absorbed dose to different lesions can be calculated and the therapy planned based on imaging data.

Individually, CAR-T cells or TRT alone may not completely cure the patient of cancer, justifying an effort for combination of the two therapies. TRT and CAR T therapies possess unique features that require special consideration when deter-mining how to dose, time, and sequence combination treatments, including the distribution of TRT dose in the body, the decay rate of the radionuclide, and the proliferation and persistence of the CAR-T cells. These characteristics complicate additive or synergistic effects of combination therapies and warrant a mathematical treatment which includes these dynamics in relation to the proliferation and clearance rates of the target tumor cells.

We have previously published mathematical models for prediction of tumor response to CAR-T cell therapy in glioma [8], the Chimeric Antigen Receptor t-cell treatment Response in GliOma (CARRGO) and for targeted radionuclide therapy [9] in a preclinical multiple myeloma disease model. The purpose of this work is to combine two previously published mathematical models of CAR-T cell and TRT therapies and use the combined, and experimentally informed, model to explore the impact of dose, timing, and sequencing of these two therapies on tumor growth.

\section{Materials and Methods}

\subsection{Mathematical model}

Mathematical models of tumor response to TRT and CAR-T cell therapies [10]have been described earlier $[8,9,11]$. The structure of the combined model is illustrated in Figure $1 \mathrm{~B}$. 
A

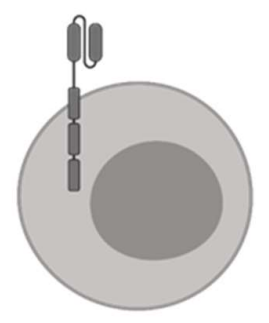

CAR T-cells

Chimeric Antigen Receptor T-cells

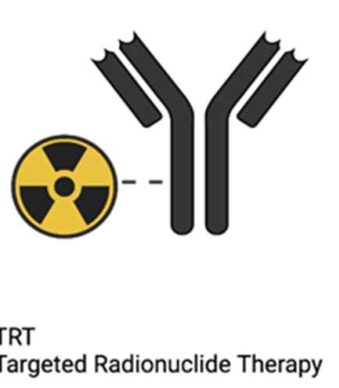

B

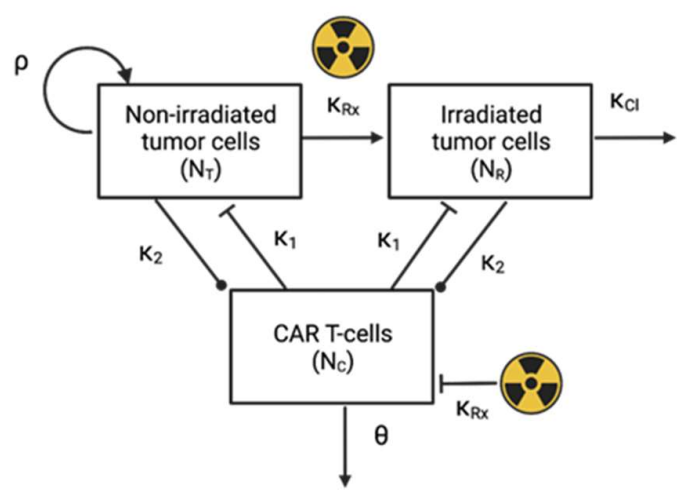

Figure 1. (A) Schematic diagram of CAR-T cells and TRT with antibody targeting. (B) Diagram of the mathematical model illustrating relationships and interactions between non-irradiated, irradiated tumor cells, TRT, and CAR-T cells.

The mathematical model assumes tumor cells are either irradiated $\left(\mathrm{N}_{\mathrm{T}}\right)$ or non-irradiated (NR) so that the total number of tumor cells is given by $T=N_{T}+N_{R}$, and that CAR$\mathrm{T}$ cells $(\mathrm{Nc})$ may also be irradiated. CAR-T cells may kill either irradiated or non-irradiated tumor cells at rate $\boldsymbol{k}_{\mathbf{1}}$ and may be stimulated to proliferate or to become exhausted upon encounter with a tumor cell at rate $\boldsymbol{k}_{2}$ and are assumed to die at a rate $\boldsymbol{\theta}$. The rate at which tumor cells or CAR-T cells become irradiated by TRT, given by $\boldsymbol{k}_{\boldsymbol{R} x}$, is modeled with the linear-quadratic equation with the Lea-Catcheside dose protraction factor [10] to account for radioactive decay of the radionuclide $\left(\lambda_{p}\right)$ and to translate absorbed radiation dose in units $\mathrm{Gy}=\mathrm{J} / \mathrm{kg}$ to a fraction of cells irradiated. Non-irradiated tumor cells grow exponentially with proliferation rate $\boldsymbol{\rho}$, which is a net rate of birth minus death rates. Irradiated tumor cells do not proliferate and are cleared out of the system at rate $\boldsymbol{k}_{\boldsymbol{c l}}$. In this work, we model alpha particle emitting TRT, for example ${ }^{225} \mathrm{Ac}$ based radionuclides. Thus, the quadratic term in Equation 4 is set to zero (i.e. $\boldsymbol{\beta}=\mathbf{0}$ ) to model high linear-energy transfer (LET) alpha-particle based radiation. The initial dose rate is calculated as $\boldsymbol{R}_{\mathbf{0}}=$ $\boldsymbol{\eta} \boldsymbol{A}_{\mathbf{0}}$ where $\boldsymbol{\eta}$ is a constant for conversion of injected activity $\boldsymbol{A}_{\mathbf{0}}$ to initial dose rate [9]. Mathematically, a treatment is "turned on" or off with the Heaviside function $\boldsymbol{H}(\boldsymbol{t}-\boldsymbol{\tau})$ which takes value zero for $\boldsymbol{t}<\boldsymbol{\tau}$ before the start of treatment and unity for $\boldsymbol{t} \geq \boldsymbol{\tau}$ during and after treatment. The parameters and values in the model are given in Table 1.

$$
\begin{aligned}
& \frac{d N_{T}}{d t}=\rho N_{T}-H\left(t-\tau_{T R T}\right) k_{R x_{-}} N_{T}-H\left(t-\tau_{C A R T}\right) k_{1} N_{T} N_{C} \\
& \frac{d N_{R}}{d t}=H\left(t-\tau_{T R T}\right) k_{R x_{-} T} N_{T}-H\left(t-\tau_{C A R T}\right) k_{1} N_{R} N_{C}-k_{c l} N_{R} \\
& \frac{d N_{C}}{d t}=k_{2}\left(N_{T}+N_{R}\right) N_{C}-H\left(t-\tau_{T R T}\right) k_{R x_{-} C} N_{C}-\theta N_{C} \\
& k_{R x_{-} T}=\alpha_{T} R_{0} e^{-\lambda_{p} t}+\frac{2 \beta R_{0}^{2}}{\gamma-\lambda_{p}}\left(e^{-2 \lambda_{p} t}-e^{-\left(\lambda_{p}+\gamma\right) t}\right) \gamma \lambda_{p}
\end{aligned}
$$


Table 1. Symbols, values, and references for parameters in the mathematical model.

\begin{tabular}{|c|c|c|c|}
\hline Parameter & Symbol & $\begin{array}{l}\text { Baseline } \\
\text { value }\end{array}$ & $\begin{array}{l}\text { Reference / } \\
\text { Comments }\end{array}$ \\
\hline $\begin{array}{l}\text { Decay constant for }{ }^{225} \mathrm{Ac} \\
\text { (1/day) }\end{array}$ & $\lambda_{\mathrm{p}}$ & 0.07 & Physical constant \\
\hline $\begin{array}{l}\text { Tumor proliferation } \\
\text { rate }(1 / \text { day })\end{array}$ & $\rho$ & 0.27 & $\begin{array}{l}\text { Mean value obtained } \\
\text { from untreated controls }\end{array}$ \\
\hline $\begin{array}{l}\text { Clearance rate of irradiated } \\
\text { tumor cells ( } 1 / \text { day })\end{array}$ & $k_{c l}$ & $\begin{array}{c}0.5 \\
\text { (held } \\
\text { constant) }\end{array}$ & [9] \\
\hline $\begin{array}{l}\text { CAR-T cell killing } \\
\text { rate }(1 / \text { day/cell })\end{array}$ & $k_{1}$ & $4.49 \times 10^{-7}$ & Optimized from data \\
\hline $\begin{array}{l}\text { CAR-T cell proliferation / } \\
\text { exhaustion rate (1/day/cell) }\end{array}$ & $k_{2}$ & $3.6 \times 10^{-13}$ & Optimized from data \\
\hline CAR-T cell death rate ( 1 /day) & $\theta$ & 0.042 & $\begin{array}{l}\text { Experimental data and optimization. } \\
\text { Range obtained from data is } 1-12 \% \text {. }\end{array}$ \\
\hline $\begin{array}{l}\text { Tumor cell } \\
\text { radiosensitivity }(1 / \mathrm{Gy})^{*}\end{array}$ & $\alpha_{\mathrm{T}}$ & 1.5 & [9] \\
\hline $\begin{array}{l}\text { CAR-T cell } \\
\text { radiosensitivity }(1 / \mathrm{Gy})^{*}\end{array}$ & $\alpha c$ & 1.5 & $\begin{array}{l}\text { Assumed equal to tumor } \\
\text { radiosensitivity }\end{array}$ \\
\hline $\begin{array}{l}\text { Activity to dose conversion } \\
\text { factor }(\mathrm{Gy} / \mathrm{day} / \mu \mathrm{Ci})\end{array}$ & $\eta$ & 3.48 & [9] \\
\hline
\end{tabular}

*Note that the radiosensitivity coefficient incorporates the effect of the radiobiological effectiveness of high linear energy transfer radiation as is the case in ${ }^{225} \mathrm{Ac}$ alpha particle therapy.

\subsection{Experimental design and model parametrization}

The parameters for ${ }^{225} \mathrm{Ac}$ - based TRT in the model $\left(\boldsymbol{\lambda}_{\boldsymbol{p}}, \boldsymbol{k}_{\boldsymbol{c l}}, \boldsymbol{\alpha}_{\{\boldsymbol{T}, \boldsymbol{C}\}}, \boldsymbol{\eta}\right)$ were derived in our earlier work comparing beta-emitting $\left({ }^{177} \mathrm{Lu}-\right)$ and alpha-emitting $\left({ }^{225} \mathrm{Ac}-\right)$ radionuclides in multiple myeloma [9]. Model parameters related to CAR-T cells $\left(\boldsymbol{k}_{\mathbf{1}}, \boldsymbol{k}_{\mathbf{2}}, \boldsymbol{\theta}\right)$ and the tumor growth rate $(\boldsymbol{\rho})$ were estimated experimentally with a mouse model of multiple myeloma as follows. Seven mice as a control group were followed using bioluminescence imaging (BLI) and the growth rate $(\boldsymbol{\rho})$ of tumor cells was calculated by fitting a monoexponential curve to the data. To obtain a measure of the CAR-T cell death rate $\boldsymbol{\theta}$ which is associated with CAR-T cell persistence, three additional mice were intravenously (i.v.) injected with 5 million MM1S multiple myeloma tumor cells that were engineered to express GFP and firefly luciferase [12] and subsequently with 1 million CS1-CAR-T cells (i.v.) on day 7.

CS1-specific CAR T cells were generated as previously described [12]. Briefly, leukapheresis products (PBMCs) from healthy donors were depleted of CD14 and CD45RA cells using microbeads. Subsequently, T naïve/memory population $(\mathrm{Tn} / \mathrm{mem})$ characterized by CD62L+ and CD45RO+ cells were enriched from the depleted population using autoMACS. The Tn/mem cells were then activated using CD3/CD28 microbeads and transduced with second generation CAR lentivirus consisting of CS1-scFv, IgG4-hinge region, 41BB costimulatory domain, and CD3z signaling domain with a truncated human EGFR domain. Following transduction, cells were maintained with IL-2 and IL-15 cytokines and expanded for 18-20 days before use. On day 28 following treatment, mice were 
sacrificed, and bone marrow samples were obtained and analyzed using flow cytometry after staining with antibodies against human CD45, CD3 and EGFR (CAR). The number of CAR-T cells and tumor cells in the samples were quantified and the percentage of CART cells compared the tumor cells (GFP+) was calculated to yield a rough estimate of the parameter $\theta$ on day 35. BLI data reflecting tumor burden on day 6 and the tumor growth rate $(\rho)$ was used to back calculate tumor burden on day 0 and scale BLI data to number of tumor cells. CAR-T cell model parameters $\boldsymbol{k}_{\mathbf{1}}, \boldsymbol{k}_{\mathbf{2}}, \boldsymbol{\theta}$ were optimized by the fitting the model to the average BLI signal of all mice treated with CAR-T cells over time. In addition to the BLI data, the estimate of $\theta$ on day 35 obtained earlier was used as a data point for optimization.

\subsection{Mathematical model simulations and analysis}

The mathematical model was implemented as follows: 5 million tumor cells are first inoculated in silico at $\mathrm{t}=0$ and proliferate untreated until day 7 , at which point either TRT or CAR-T cell therapy is simulated so that the initial conditions are $\boldsymbol{N}_{T}(\boldsymbol{t}=\mathbf{0})=\mathbf{5} \times \mathbf{1 0}^{\mathbf{6}}$ and $\boldsymbol{N}_{\boldsymbol{R}}(\boldsymbol{t}=\mathbf{0})=\mathbf{0}$. The impact of therapy was evaluated with three metrics of tumor growth- progression free survival (PFS), overall survival (OS) and time to nadir, or minimum tumor burden post-therapy $\left(t_{\min }\right)$. PFS was defined as the last time point where the tumor volume exceeded the tumor volume prior to therapy on day 7. OS was defined as the day the tumor reached $10^{11}$ cells. Four treatment regimens were evaluated with the mathematical model simulations 1) TRT only, 2) CAR-T cells only, 3) CAR-T cell therapy followed by TRT and 4) TRT followed by CAR-T cell therapy. The interval between the therapies is varied and maximum PFS, OS and $t_{\text {min }}$ for these therapeutic regimens as well as the optimal interval between therapies were investigated.

To evaluate the sensitivity of the model to the model parameters and therapeutic doses, each of these parameters (TRT injected activity, CAR-T dose, tumor burden, $\left.\boldsymbol{\rho}, \boldsymbol{k}_{1}, \boldsymbol{k}_{2}, \boldsymbol{\theta}, \boldsymbol{\alpha}_{\boldsymbol{c}}\right)$ were changed by $\pm 50 \%$ and the maximum PFS, OS and $t_{\text {min }}$ were calculated. Based on this analysis the most important parameters influencing the outcome were determined. Lastly, PFS and OS were calculated by varying the parameter of highest sensitivity and the implication for optimizing the combination therapy.

\section{Results}

\subsection{Parameters for CAR T treatment model}

Figure 2A shows the number of CAR-T cells, tumor cells as well as percentage of CAR-T cells (Figure 2B) against tumor cells obtained from the mice tumor samples on day 28 . The percent of CAR-T cells on day 28 to tumor cells range from $1 \%$ and $12 \%$. Figure $2 \mathrm{C}$ shows the fit of tumor growth curve to the untreated mice BLI tumor burden data. The simulated tumor burden fit to the CAR-T mice experiment data (Figure 2C). 
A

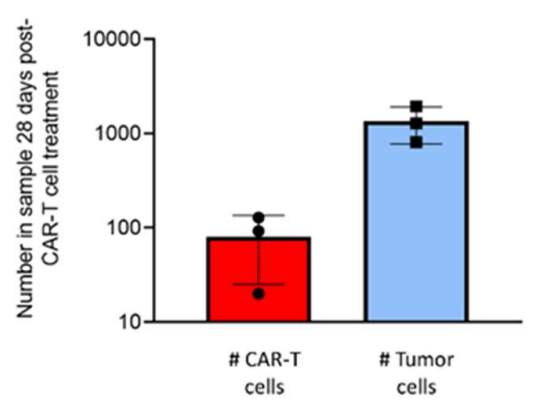

C

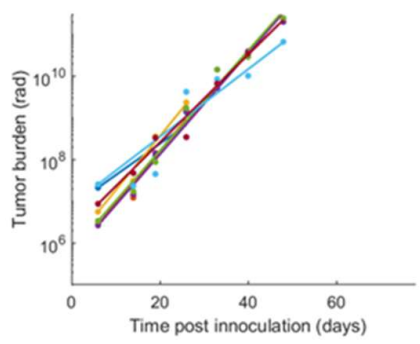

B

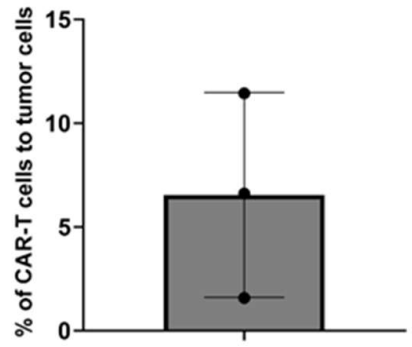

D

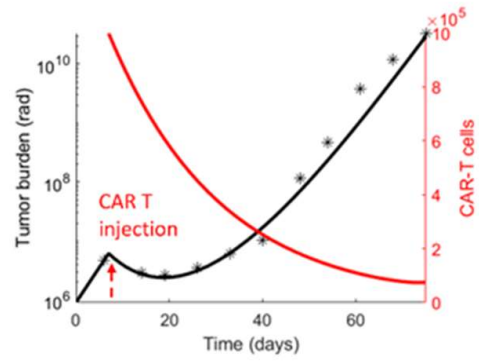

Figure 2. CAR-T cell data and model parameters. (A) Estimation of CAR-T cell persistence $(\boldsymbol{\theta})$. Three mice were sacrificed and (B) percentage of CAR-T cells to tumor cells on day 35 were used to estimate the CAR-T cell death rate $\theta$ (1/time). (C) Tumor burden as measured with BLI in radiance (rad) for untreated control mice $(\mathrm{N}=7)$ following administration of 1 million MM1S multiple myeloma cells at time $\boldsymbol{t}=\mathbf{0}$ to estimate the net tumor growth rate $(\rho)$. (D) CAR-T cell killing $\left(\boldsymbol{k}_{\mathbf{1}}\right)$ and proliferation/exhaustion $\left(\boldsymbol{k}_{2}\right)$ parameters are estimated by fitting the mathematical model to BLI data $\left({ }^{* \prime} \mathrm{s}\right)$ from mice treated with CAR-T cells on day $7(\mathrm{~N}=3)$.

A

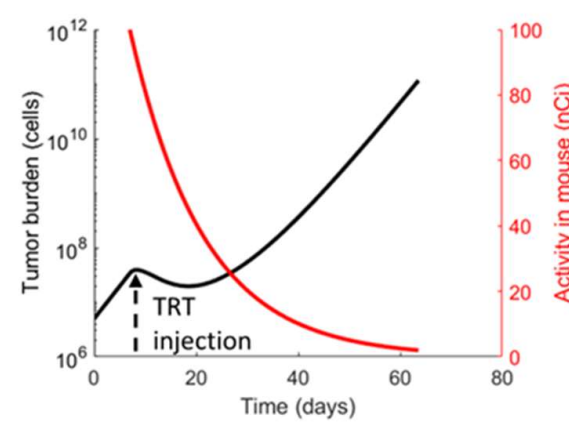

C

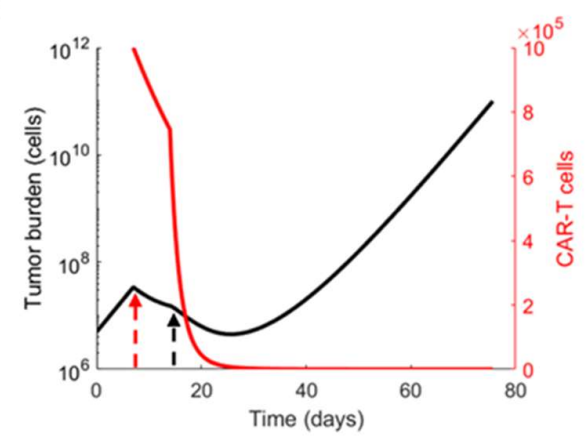

B

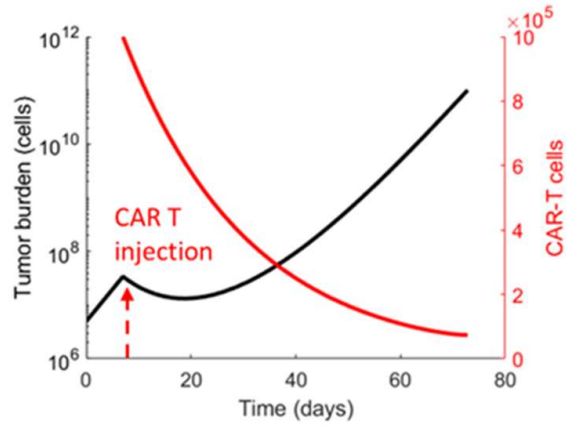

D

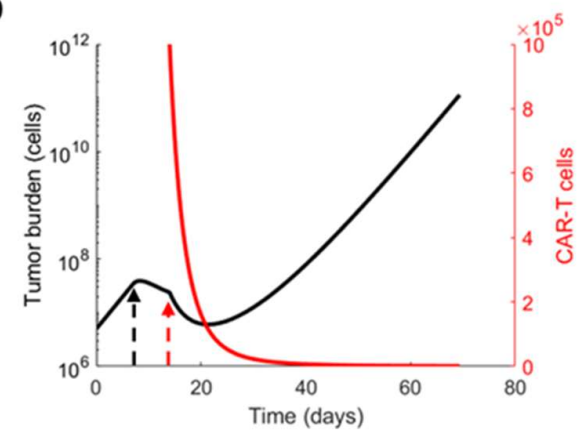


Figure 3. Model simulations of TRT and CAR T therapy alone and in combination. Mono therapy simulations of (A) TRT treatment (black arrow, $\mathrm{A}_{0}=100 \mathrm{nCi}$ ) or (B) CAR-T cell (red arrow, $\boldsymbol{N}_{\boldsymbol{C}}(\boldsymbol{t}=\mathbf{7})=\mathbf{1} \times \mathbf{1 0}^{\mathbf{6}}$ ) administered on day $\boldsymbol{t}=\mathbf{7}$. Combination treatment simulations with (C) CAR-T cells on day $\boldsymbol{t}=\mathbf{7}$ followed by TRT on day 14 and (D) TRT on day 7 followed by CAR-T cells on day 14. The mathematical model predicts tumor growth, radionuclide decay, and CAR-T cell populations over time.

\subsection{Evaluating therapeutic regimens}

CAR-T cell immunotherapy and targeted radionuclide therapies either as monotherapies or combination therapies were simulated in silico with the mathematical model (Figure 3). Reduced tumor burden immediately post therapy (day 7) is seen in response to TRT (Figure 3A) or CAR-T therapy (Figure 3B) or combination of the two therapies when TRT is given 1 week post CAR-T therapy (Figure 3C) or CAR-T therapy is given 1 week post TRT (Figure 3d). Sensitivity of CAR-T cells to TRT results in shorter persistence of CAR-T cells when TRT is given since TRT can kill CAR-T cells (Figure 3D). When a second therapy is given on day 14 as combination therapy regimen (Figure 3C, D), the model predicts several important effects that are independent of the therapy sequence. Two inflections in the tumor burden curve are evident and the minimum tumor burden in both cases is lower than that obtained by monotherapy alone, showing an additive effect of combination therapy. The time to nadir in tumor burden is also increased along with the increase in progression-free and overall survival (Table 2). Simulations with experimentally derived model parameters (Table 1) shows that the duration of tumor response (PFS and OS) is prolonged with the CAR-T dose of 1 million cells as compared to TRT injected activity of $100 \mathrm{nCi}$. Table 2 shows the time to minimum tumor burden, progression free survival or overall survival for each treatment scenario.

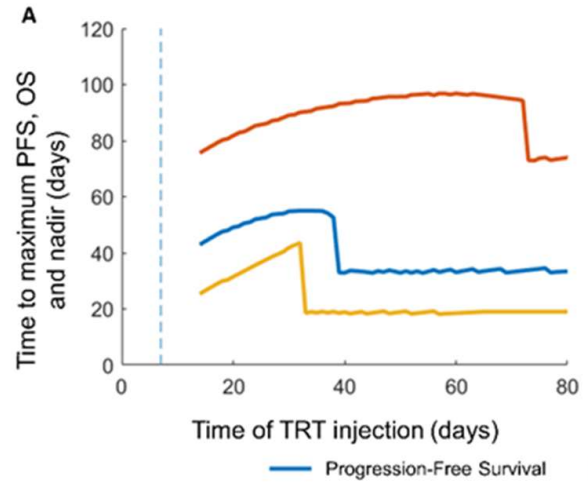

(A) CAR-T cell therapy prior to TRT

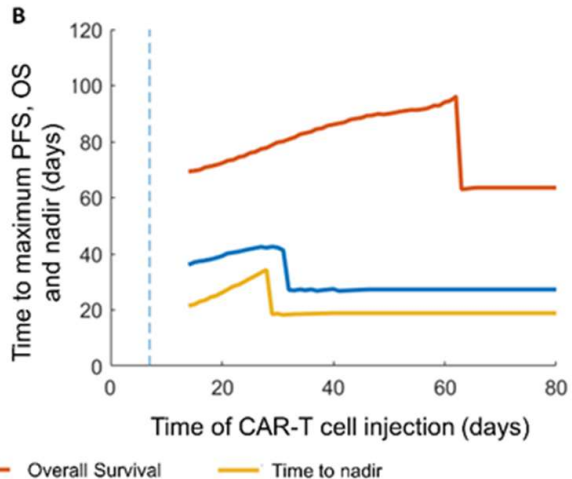

(B) TRT prior to CAR-T cell therapy

Figure 4. Simulated response to combination TRT and CAR-T cell therapies. Progression free survival (PFS), overall survival (OS), and time to nadir for two treatment sequences: (A) CAR-T cells on day $t=7$ followed by TRT starting from $t=14-80$. A clear maximum benefit is seen in PFS, OS and time to nadir. (B) TRT on day $t=7$ followed by CAR $T$ starting from $t=14-80$. Time to maximum OS, PFS and nadir is measured from when the tumor is initiated at $t=0$.

\subsection{Sequence of therapy and maximizing survival}

Figure 4 shows the dependence of progression-free survival, overall survival and time to tumor burden nadir when comparing the sequence of therapies with either CAR$\mathrm{T}$ cells as the first therapy (Figure 4A) or TRT as the first therapy (Figure 4B). An improvement in both PFS and OS is predicted when CAR-T cells are given prior to TRT. The increased killing of CAR-T cells due to radiation which is still present post-week 2 due to the long radionuclide half-life, is the reason for reduced PFS when TRT precedes CAR-T 
cell therapy. While a maximum in each of the curves is seen, the curve for overall survival shows that there is a range of timing the second therapy that can yield overall survival very close to the maximum value. The abrupt drop in OS means that there is a point in time after which there is no benefit to giving a second therapy, regardless of the ordering of the two therapies. The model predicts that with the experimentally obtained parameters for the current system, CAR-T cell therapy given prior to TRT will increase PFS, OS, and time to tumor burden nadir as compared to TRT given prior to CAR-T cell therapy (Table 2).

Table 2. Simulated measures of tumor response to individual and combination therapies. First therapy is given 7 days post tumor initiation. For combination therapy, the second therapy is given 7 days following the first therapy.

\begin{tabular}{|c|c|c|c|c|c|}
\hline Response criteria & Control & $\begin{array}{c}\text { TRT } \\
\text { Only (day 7) }\end{array}$ & $\begin{array}{c}\text { CAR T } \\
\text { Only (day 7) }\end{array}$ & $\begin{array}{l}\text { CAR T } \\
\text { Before } \\
\text { TRT }\end{array}$ & $\begin{array}{c}\text { TRT } \\
\text { Before } \\
\text { CAR T }\end{array}$ \\
\hline $\begin{array}{c}\text { Progression-Free Sur- } \\
\text { vival } \\
\text { (PFS) (days) }\end{array}$ & - & 27 & 33 & 55 & 43 \\
\hline Overall Survival (days) & 43 & 64 & 73 & 97 & 96 \\
\hline Time to nadir (days) & - & 19 & 19 & 44 & 34 \\
\hline $\begin{array}{l}\text { Interval between thera- } \\
\text { pies } \\
\text { for maximizing PFS } \\
\text { (days) }\end{array}$ & - & - & - & 25 & 22 \\
\hline
\end{tabular}




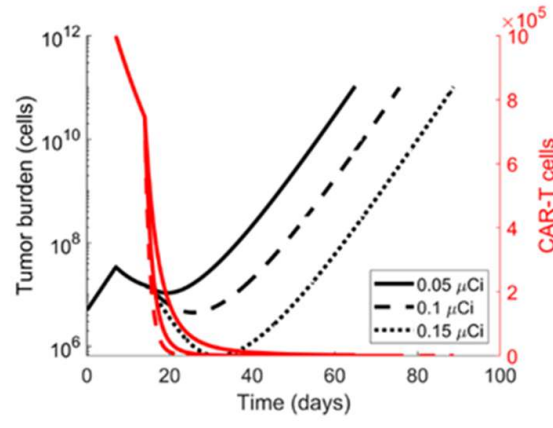

(A) Injected TRT activity $\left(A_{0}\right)$

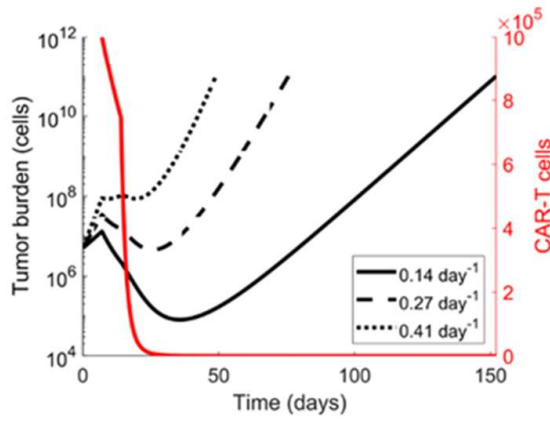

(D) Tumor proliferation rate $(\rho)$

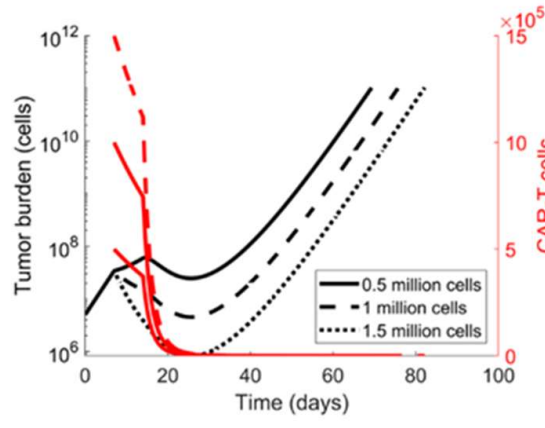

(B) CAR-T cell dose

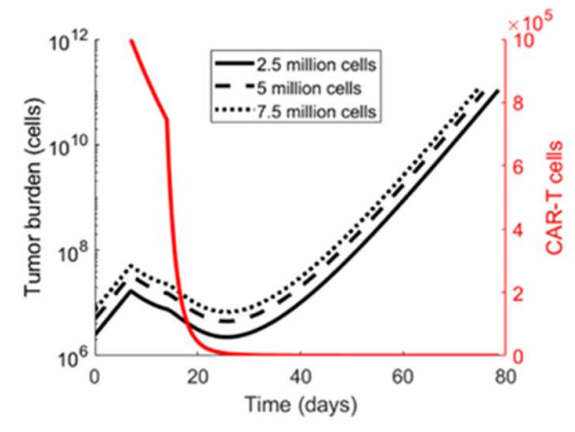

(C) Tumor Burden

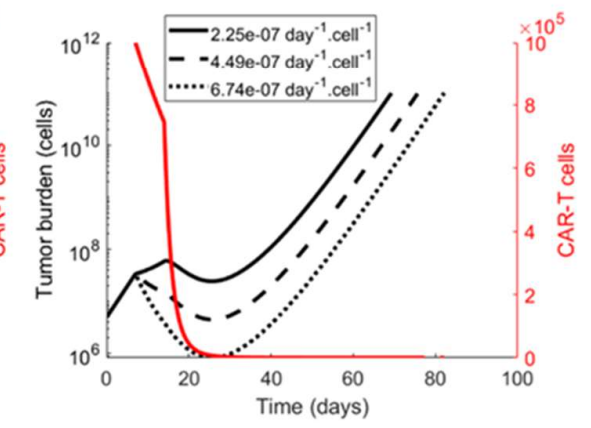

(E) CAR T-cell killing rate $\left(k_{1}\right)$

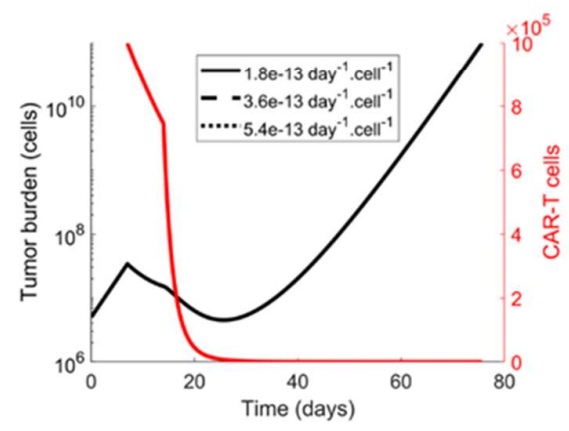

(F) CAR-T cell proliferation rate $\left(k_{2}\right)$

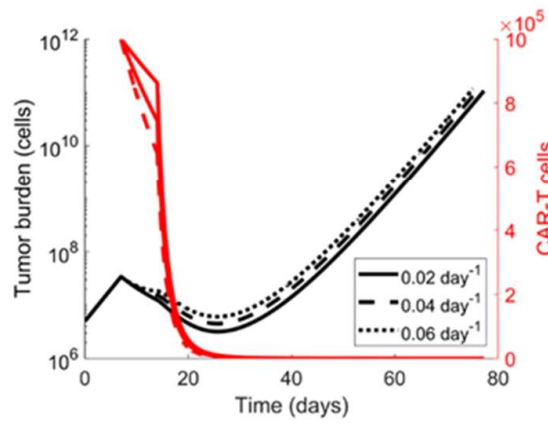

(G) CAR-T cell death rate $(\theta)$

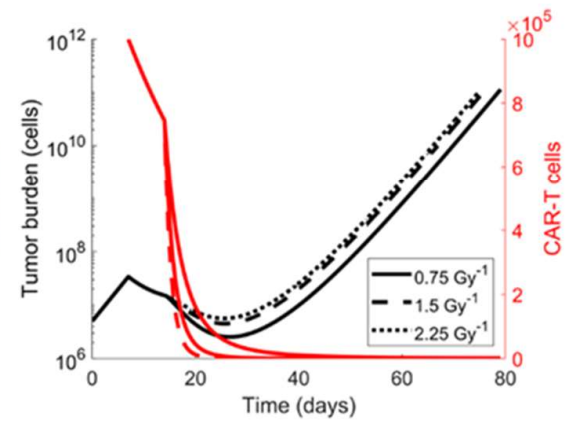

(H) CAR-T radiosensitivity $\left(\alpha_{c}\right)$

Figure 5. Sensitivity analysis. Impact of model parameter variation (+/-50\%) on tumor growth curves for CAR $\mathrm{T}$ (day $\mathrm{t}=7$ ) followed by TRT (day $t=14$ ) therapy. Variation in tumor proliferation has the largest impact on tumor growth response to combination therapy.

\subsection{The impact of model parameters and TRT-CAR-T cell combination therapy on tumor growth}

To examine the sensitivity of the model predictions to variations in the parameters, each parameter was changed independently by $+/-50 \%$ and a simulation of combination therapy of CAR T on day 7 followed by TRT on day 14 were performed (Figure 5). The parameter with the greatest effect on the tumor growth rate $(\rho)$ while the parameter with least influence was the CAR-T cell proliferation and exhaustion rate $\mathrm{k}_{2}$. The value of $\mathrm{k}_{2}$ estimated from the data (Figure 2D) was extremely small and thus its impact on tumor growth dynamics was also small. In all scenarios, the model predicts that the population of CAR-T cells precipitously drops following administration of TRT. Thus, the prediction 
is that the therapeutic advantage of CAR-T cells in a combination therapy comes prior to administration of TRT due to the effect of radiation on the CAR-T cells.

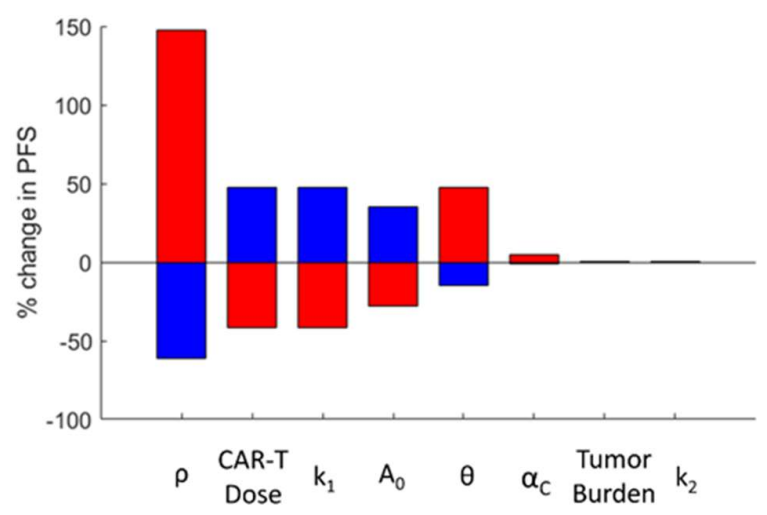

(A) PFS

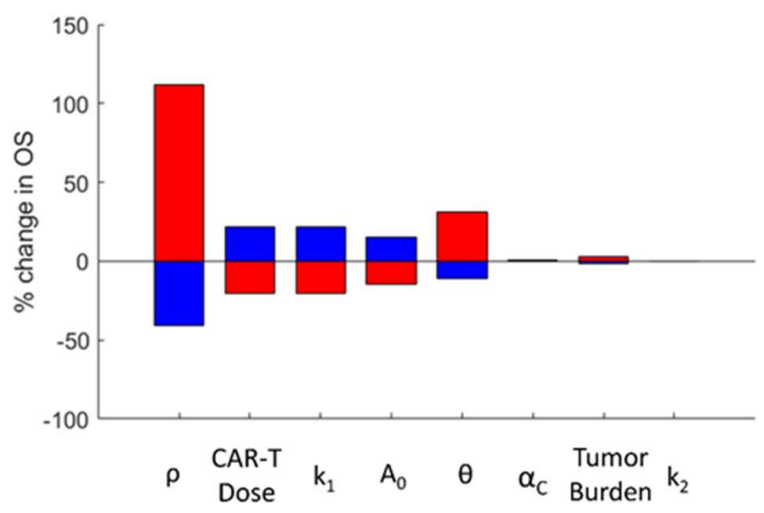

(B) OS

Figure 6. Sensitivity study of model parameters on survival outcomes. Change in Progression-Free Survival (A), and overall survival (B) is shown for $+/-50 \%$ change in each of the model parameters. Blue indicates $+50 \%$ change and red indicates $50 \%$ change in the parameter. Variations in the tumor proliferation rate have the largest impact on PFS and OS.

Figure 6 summarizes the impact of model and therapeutic parameters on predicted PFS and OS. The tumor proliferation rate has the greatest impact on PFS and OS. Using the experimentally derived model parameters, CAR-T dose is predicted to have a slightly greater impact than TRT on OS and PFS. CAR-T cell radiosensitivity has a greater impact on PFS than OS since the curve for OS is relatively flat over a large range of therapeutic intervals. Conversely, changes in initial tumor burden impacts OS but does not impact PFS since the tumor dynamics are similar between the two cases and because PFS is a relative measurement from the start of the therapy. Changes in CAR-T cell dose, TRT dose, CAR-T cell killing rate $\mathrm{k}_{1}$, and proliferation/exhaustion rate $\mathrm{k}_{2}$ were directly proportional to changes in PFS and OS, however an inverse relationship was observed for tumor proliferation rate $\rho$, CAR-T cell persistence $\theta$, tumor burden and radiation sensitivity of CAR$\mathrm{T}$ cells $\alpha$ c.

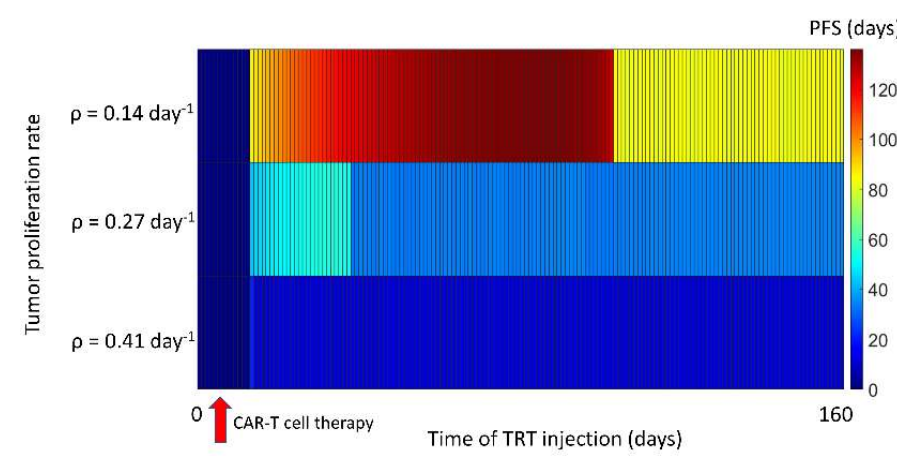

(A) PFS

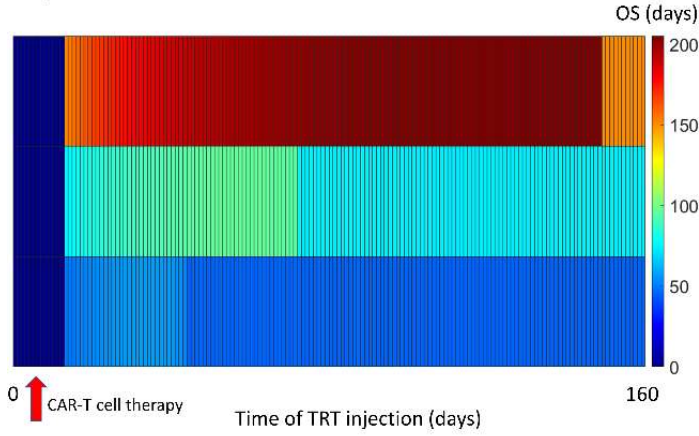

(B) OS

Figure 7. Impact of tumor proliferation rate on PFS and OS tumor growth. (A) PFS (B) OS. Paradoxically, a faster growing tumor results in lower PFS and OS due to a greater response to the treatments. This suggests that the interval between the therapies should be smaller for faster growing tumors. 
Based on the results of the sensitivity study which demonstrated tumor proliferation as the most important factor influencing PFS and OS, we examined the effect of low, medium, and high tumor proliferation rates on PFS and OS as a function of the time of TRT injection following CAR-T cell therapy (Figure 7). Interestingly, if the tumor growth rate is high, then the PFS and OS are relatively lower due to the increased response to the treatments. However, what is also evident is that the optimal day of administration of the second therapy is also different. The interval between therapies for tumors that grow faster needs to be reduced as compared to a slower growing tumor.

\section{Discussion}

Here we have presented a mathematical model combining CAR-T cell immunotherapy and targeted radionuclide therapies for the treatment of cancer with application to multiple myeloma as an example. The proposed model combines our previously developed models for CAR-T therapy (CARRGO model in [8]) and ${ }^{225}$ Ac-DOTA-daratumumab targeted radionuclide therapy [9]. The model predicts that it is feasible to optimize the dose and timing of the two therapies to maximize tumor growth delay. Using model parameters derived from experimental data predict a better survival outcome when CAR-T cells are given prior to TRT. However, different disease or therapy combinations might result in different combinations of parameters and potentially different predictions. Thus, it is important to assess disease or therapy-specific parameters.

A key result of the model is the time interval between the two therapies should be modified based on the proliferation rate of the tumor. Thus, measurement of growth rate of different kinds of cancers can help in optimization of the combination therapy. While the model has been applied to a setting where the immunotherapeutic is the CS1 CAR-T cell and the radiation therapy is provided by targeted deliver of ${ }^{225}$ Ac-DOTA-Daratumumab to CD38 receptors in multiple myeloma, the model can be applied to general immunotherapeutic and TRT combinations with various targets and therapeutics. Example can be targeting the BCMA CAR-T cells [13-15] instead of CS1 CAR-T cells or targeting with a beta particle therapeutic like ${ }^{177} \mathrm{Lu}$ rather than alpha particle therapeutic like ${ }^{225} \mathrm{Ac}$.

The mathematical formulation in the proposed model makes assumptions that may be disease and application specific. The simplifying assumption of exponential tumor growth is consistent with experimental preclinical data presented here, however, tumor growth rates evaluated at later time points can slow down, reflecting the sigmoidal growth. Clinically, tumors can grow slower than preclinical models where the assumption of exponential growth rate would suffice. An important aspect to note of the model is the mass-action kinetics of CAR-T cell killing $\left(\boldsymbol{k}_{\mathbf{1}}\right)$ and proliferation/exhaustion $\left(\boldsymbol{k}_{\mathbf{2}}\right)$, that permits oscillating solutions which are not realistic or likely to be observed in vivo. We note that consistent with our prior work in this model[8], the observed parameter ranges do not predict oscillating solutions.

Additionally, here we assume a mono-exponential decay of CAR-T cells, however, there is some evidence of a bi-exponential decay in the CAR-T cell concentration in the blood [16]. A key reason for this assumption is that here we use the CAR-T cell percentage measured in the bone marrow rather than the blood. In this scenario, the magnitude of the exponent of the mono-exponential decay will be higher, dominating over a bi-exponential dynamic. Moreover, it is assumed in the current work that the CAR-T cells are well mixed and evenly distributed with the tumor cells. Of course, CAR-T cells can distribute across different organs of the body, potentially increasing the number of CAR-T cells in the tumor. The distribution of the CAR-T cells can also be variable across the tumor and different CAR-T cell densities can result in variable response across the tumor. Although the well-mixed assumption is reasonable for a disseminated disease such as multiple myeloma, repeated measurements of CAR-T cells in the tumor in a preclinical model setting would help support this assumption. In our experimentally derived parameters, the value of $\mathrm{k}_{2}$ which indicates CAR-T cell proliferation or exhaustion is extremely low as compared to killing rate constant $\mathrm{k}_{1}$, indicating very low proliferation of CAR-T cells - thus the CAR- 
$\mathrm{T}$ cell numbers in the body follow a mono-exponential decay curve. However, this might not be the general case. In two lesions evaluated using the CARRGO model[8], the value of $\mathrm{k}_{2}$ in a lesion with favorable response to CAR-T cell therapy was significantly higher than a non-responding lesion indicating more complex immune system dynamics.

A critical issue to consider in combination therapies involving immune and radiation therapies is toxicity. Toxicity in immunotherapy is typically shown in the form of cytokine release syndrome that limits the dose of therapeutic administered. On the other hand, radiation toxicity tends to be proportional to dose in peripheral organs, mostly the bone marrow. Apart from individual therapeutic toxicities, the mechanistic interactions between radiation and immunotherapy and potential common targets of both therapies can pose limits to either of the two therapies and these cells or organs might need to be modeled into the mathematical framework to optimize the dosage, interval and number of cycles either of these therapies can be administered. Additionally, there is recent evidence that radiation can induce changes in the immune system and can stimulate significant immune response for better therapeutic efficacy [17-19]. With a better understanding of the mechanistic basis and experimental data to support, the interactions between radiation and immunotherapy can be better modeled and additional interaction terms can be introduced in the mathematical formulation to account for toxicity. With the current formulation, the effect of radiation results in death of CAR-T cells, thus it is advantageous to administer CAR-T cells before TRT; however, with the stimulation of the immune system with radiation and subsequent expansion of the model for radiation-immune interactions, TRT before immunotherapy might present a better therapeutic outcome for survival. The CAR-T cells that are stimulated by radiation can then be separately modeled in the mathematical framework and a result in increased tumor eradication.

\section{Conclusion}

With increasing number of therapies and possible combination of therapies, it has become essential to incorporate mathematical models to consider the effects of dose, sequence, and timing of multiple therapies. Here we investigated a mathematical model of CAR-T cell immunotherapy and targeted radionuclide therapy. The mathematical model calibrated with experimental data makes two predictions about this therapeutic combination; 1) tumor proliferation rate is the most important factor in determining the timing between the therapies and 2) CAR-T cells followed by TRT will be more efficacious than TRT followed by CAR T. These predictions are specific to the disease model (MM1S multiple myeloma), CAR-T cells (CS1), and TRT (225Ac-dota-daratumumab) therapeutic modalities investigated here, however it is possible these predictions may be generalized and applied to other disease settings. Additional studies are warranted to investigate combination therapies with multiple administrations of one or more of the treatments.

Supplementary Materials: The following are available online at www.mdpi.com/xxx/s1, Figure S1, S2 and S3, Table S1, S2, Video S1, S2, Supplementary data table on BLI of control and CAR-T cell treated mice.

Author Contributions: Conceptualization, V.A. and R.R.; methodology, V.A., R.R.; software, NA; validation, V.A., D.A., A.B., E.C., A.K., F.P., M.M., J.S., J.W., X.W., R.R.; formal analysis, V.A., D.A., A.B., E.C., A.K., F.P., M.M., J.S., J.W., X.W., R.R.; investigation, V.A., D.A., E.C., F.P., M.M., J.S., X.W., R.R.; resources, X.X.; data curation, D.A., E.C., M.M; writing-original draft preparation, V.A., R.R.; writing-review and editing, V.A., D.A., A.B., E.C., F.P., M.M., J.S., J.W., X.W., R.R.; visualization, NA; supervision, J.S., X.W., F.P., R.R.; project administration, J.S., X.W., F.P., R.R.; funding acquisition, J.S., X.W., F.P., R.R. All authors have read and agreed to the published version of the manuscript.

Funding: Research reported in this publication was supported by the National Cancer Institute of the National Institutes of Health under grants R01CA238429 (R.R., J.S., X.W., F.P.) and P30CA03357 City of Hope Comprehensive Cancer Center. 
Data Availability Statement: Supplementary data table on BLI of control and CAR-T cell treated mice are provided.

Acknowledgments: The authors acknowledge support from the Judy and Bernard Briskin Center.

Conflicts of Interest: The authors declare no conflict of interest. The funders had no role in the design of the study; in the collection, analyses, or interpretation of data; in the writing of the manuscript, or in the decision to publish the results.

\section{References}

1. Esfahani, K., et al., A review of cancer immunotherapy: from the past, to the present, to the future. Curr Oncol, 2020. 27(Suppl 2): p. S87-S97, doi: $10.3747 /$ co.27.5223.

2. Wang, Y., et al., Combining Immunotherapy and Radiotherapy for Cancer Treatment: Current Challenges and Future Directions. Front Pharmacol, 2018. 9: p. 185, doi: 10.3389/fphar.2018.00185.

3. Formenti, S.C. and S. Demaria, Future of Radiation and Immunotherapy. Int J Radiat Oncol Biol Phys, 2020. 108(1): p. 3-5, doi: 10.1016/j.ijrobp.2020.04.034.

4. Hyrenius-Wittsten, A. and K.T. Roybal, Paving New Roads for CARs. Trends Cancer, 2019. 5(10): p. 583-592, doi: 10.1016/j.trecan.2019.09.005.

5. Waldman, A.D., J.M. Fritz, and M.J. Lenardo, A guide to cancer immunotherapy: from T cell basic science to clinical practice. Nat Rev Immunol, 2020. 20(11): p. 651-668, doi: 10.1038/s41577-020-0306-5.

6. Sgouros, G., et al., Radiopharmaceutical therapy in cancer: clinical advances and challenges. Nat Rev Drug Discov, 2020. 19(9): p. 589608, doi: 10.1038/s41573-020-0073-9.

7. St James, S., et al., Current Status of Radiopharmaceutical Therapy. Int J Radiat Oncol Biol Phys, 2021. 109(4): p. 891-901, doi: 10.1016/j.ijrobp.2020.08.035.

8. Sahoo, P., et al., Mathematical deconvolution of CAR T-cell proliferation and exhaustion from real-time killing assay data. J R Soc Interface, 2020. 17(162): p. 20190734, doi: 10.1098/rsif.2019.0734.

9. Minnix, M., et al., Comparison of CD38-Targeted alpha-Versus beta-Radionuclide Therapy of Disseminated Multiple Myeloma in an Animal Model. J Nucl Med, 2021. 62(6): p. 795-801, doi: 10.2967/jnumed.120.251983.

10. Brenner, D.J., et al., The linear-quadratic model and most other common radiobiological models result in similar predictions of time-dose relationships. Radiat Res, 1998. 150(1): p. 83-91.

11. Neira, S., et al., A kinetic model of continuous radiation damage to populations of cells: comparison to the LQ model and application to molecular radiotherapy. Phys Med Biol, 2020. 65(24): p. 245015, doi: 10.1088/1361-6560/aba21d.

12. Wang, X., et al., Lenalidomide Enhances the Function of CS1 Chimeric Antigen Receptor-Redirected T Cells Against Multiple Myeloma. Clin Cancer Res, 2018. 24(1): p. 106-119, doi: 10.1158/1078-0432.CCR-17-0344.

13. Ali, S.A., et al., $T$ cells expressing an anti-B-cell maturation antigen chimeric antigen receptor cause remissions of multiple myeloma. Blood, 2016. 128(13): p. 1688-700, doi: 10.1182/blood-2016-04-711903.

14. <Brudno_2018_JCO.pdf>. doi: $10.1200 / \mathrm{JCO}$

10.1200/JCO.2018.

15. Raje, N., et al., Anti-BCMA CAR T-Cell Therapy bb2121 in Relapsed or Refractory Multiple Myeloma. N Engl J Med, 2019. 380(18): p. 1726-1737, doi: 10.1056/NEJMoa1817226.

16. Chaudhury, A., et al., Chimeric Antigen Receptor T Cell Therapies: A Review of Cellular Kinetic-Pharmacodynamic Modeling Approaches. J Clin Pharmacol, 2020. 60 Suppl 1: p. S147-S159, doi: 10.1002/jcph.1691.

17. Bernstein, M.B., et al., Immunotherapy and stereotactic ablative radiotherapy (ISABR): a curative approach? Nat Rev Clin Oncol, 2016. 13(8): p. 516-24, doi: 10.1038/nrclinonc.2016.30.

18. Jagodinsky, J.C., P.M. Harari, and Z.S. Morris, The Promise of Combining Radiation Therapy With Immunotherapy. Int J Radiat Oncol Biol Phys, 2020. 108(1): p. 6-16, doi: 10.1016/j.ijrobp.2020.04.023.

19. Patel, R.B., et al., Low-dose targeted radionuclide therapy renders immunologically cold tumors responsive to immune checkpoint blockade. Science Translational Medicine, 2021. 13(602): p. eabb3631, doi: 10.1126/scitranslmed.abb3631. 